\title{
Editors
}

Giuseppe E. Bruno

Dipartimento Interateneo di Fisica "M. Merlin", Università e Politecnico di Bari

Via Orabona 4, 70126 Bari, Italy

e.mail: giuseppe.bruno@ba.infn.it

Gabriele Chiodini

Istituto Nazionale di Fisica Nucleare - Sezione di Lecce

Via Arnesano, 73100 Lecce, Italy

e.mail: gabriele.chiodini@le.infn.it

Pietro Colangelo

Istituto Nazionale di Fisica Nucleare - Sezione di Bari

Via Orabona 4, 70126 Bari, Italy

e.mail: pietro.colangelo@ba.infn.it

Claudio Corianò

Dipartimento di Matematica e Fisica "E. De Giorgi”, Università del Salento

Via Arnesano, 73100 Lecce, Italy

e.mail: claudio.coriano@le.infn.it

Donato M. Creanza

Dipartimento Interateneo di Fisica "M. Merlin", Università e Politecnico di Bari

Via Orabona 4, 70126 Bari, Italy

e.mail: donato.creanza@ba.infn.it

Fulvia De Fazio

Istituto Nazionale di Fisica Nucleare - Sezione di Bari

Via Orabona 4, 70126 Bari, Italy

e.mail: fulvia.defazio@ba.infn.it

Eugenio Nappi

Istituto Nazionale di Fisica Nucleare - Sezione di Bari

Via Orabona 4, 70126 Bari, Italy

e.mail: eugenio.nappi@ba.infn.it

Stefania Spagnolo

Dipartimento di Matematica e Fisica "E. De Giorgi", Università del Salento Via Arnesano, 73100 Lecce, Italy

e.mail: stefania.spagnolo@1e.infn.it 


\section{Scientific Advisory Committee}

Roberto Casalbuoni (Firenze University)

Fernando Ferroni (Roma Sapienza University and INFN)

Michelangelo Mangano (CERN Geneva)

Nello Paver (Trieste University)

Mikhail A. Shifman (Minnesota University)

George Sterman (SUNY Stony Brook)

Sheldon Stone (Syracuse University)

\section{Local Organizing Committee}

Leonardo Angelini (Bari University)

Giuseppe E. Bruno (Bari Polytechnic)

Gabriele Chiodini (INFN Lecce)

Pietro Colangelo (INFN Bari)

Claudio Corianò (Università del Salento)

Donato M. Creanza (Bari Polytechnic)

Fulvia De Fazio (INFN Bari)

Eugenio Nappi (INFN Bari)

Stefania Spagnolo (Università del Salento)

\section{Scientific organization}

Konstantinos Bachas (INFN Lecce)

Floriana Giannuzzi (Bari University)

Stefano Nicotri (INFN Bari)

Giuseppe Trombetta (Bari University) 


\section{Participants}

ABBAS, Gauhar

ABUKI, Hiroaki

ALFORD, Mark

ANGELINI, Leonardo

BACHAS, Konstantinos

BANDYOPADHYAY, Priyotosh

BARABANOV, Mikhail

BELLANTUONO, Loredana

BERAUDO, Andrea

BIGAZZI, Francesco

BIZZETI, Andrea

BRUNO, Giuseppe Eugenio

BURAS, Andrzej

CAFAGNA, Francesco Saverio

CHELABI, Kaddour

CHIODINI, Gabriele

CHIRILLI, Giovanni Antonio

COLANGELO, Pietro

CORIANÓ, Claudio

COSTANTINI, Antonio

CREANZA, Donato

D'AMBROSIO, Giancarlo

DE FAZIO, Fulvia

DELLE ROSE, Luigi

DESTEFANIS, Marco

DVOEGLAZOV, Valery

FABBRICHESI, Marco

FAZIO, Angelo Raffaele

FINI, Rosa Anna

FRAMPTON, Paul

GIANNUZZI, Floriana

GREYNAT, David

GUZZI, Marco

HUANG, Mei

IVANOV, Mikhail

KALAYDZHYAN, Tigran

KEYES, Robert

KÖNIG, Matthias

LUCHA, Wolfgang

MAMMADOV, Shahin

MANZARI, Vito
IFIC, Valencia, SPAIN

Aichi University of Education, Kariya, JAPAN

Washington University, St Louis, USA

University of Bari and INFN - Sezione di Bari, ITALY

INFN - Sezione di Lecce, ITALY

INFN - Sezione di Lecce, ITALY

JINR, Dubna, RUSSIA

University of Bari and INFN - Sezione di Bari, ITALY

INFN - Sezione di Torino, ITALY

INFN - Sezione di Pisa, ITALY

Univ. of Modena - Reggio Emilia and INFN - Firenze, ITALY

Politecnico di Bari and INFN - Sezione di Bari, ITALY

TUM- IAS, Munich, GERMANY

INFN - Sezione di Bari, ITALY

Institute of theoretical Physics, Beijing, CHINA

INFN - Sezione di Lecce, ITALY

University of Regensburg, Regensburg, GERMANY

INFN - Sezione di Bari, ITALY

University of Salento, Lecce, ITALY

University of Salento and INFN - Sezione di Lecce, ITALY

Politecnico di Bari, ITALY

INFN - Sezione di Napoli, ITALY

INFN - Sezione di Bari, ITALY

University of Southampton, Southampton, UK

University of Torino and INFN - Sezione di Torino, ITALY

Universidad de Zacatecas, Zacatecas, MEXICO

INFN - Sezione di Trieste, ITALY

Universidad Nacional de Colombia, Bogotá, COLOMBIA

INFN - Sezione di Bari, ITALY

University of North Carolina, Chapel Hill, USA

University of Bari, Bari, ITALY

University of Napoli and INFN - Sezione di Napoli, ITALY

University of Manchester, Manchester, UK

IHEP, CAS, Beijing, CHINA JINR, Dubna, RUSSIA

University of Illinois at Chicago, Chicago, USA

McGill University, Montreal, CANADA

JGU Mainz, Mainz, GERMANY

Austrian Academy of Sciences, Vienna, AUSTRIA

Baku State University, Baku, AZERBAIJAN

INFN - Sezione di Bari, ITALY 
MARGETIS, Spyridon

MARZO, Carlo

MAZUMDAR, Kajari

MAZZILLI, Marianna

MEGIAS, Eugenio

MELIKHOV, Dmitri

MENINNO, Elisa

NAPPI, Eugenio

NICOTRI, Stefano

OCALAN, Kadir

OZPINECI, Altug

PALANO, Antimo

PALESE, Marcella

PAPPAGALLO, Marco

PASQUINI, Barbara

PASTORE, Alessandra

PRELOVSEK, Sasa

QAMESH, Ahmed

REINOSA, Urko

ROMANIOUK, Anatoli

RUGGIERI, Marco

SANTOPINTO, Elena

SANTORELLI, Pietro

SCRIMIERI, Egidio

SERINO, Mirko

SHIVAJI, Ambresh

TANHA, Milad

TASSI, Enrico

TOIA, Alberica

TRELEANI, Daniele

TRENTADUE, Luca

TROMBETTA, Giuseppe

WANG, Wei

WANG, Yuming

WATANABE, Akira

WINTERROTH, Ekkehart

YAMAGUCHI, Yasuhiro

ZAMIRALOV, Valeriy
Kent State University, Kent, USA

University of Salento, Lecce, ITALY

Tata Institute of Fundamental Research, Mumbai, INDIA

University of Bari and INFN - Sezione di Bari, ITALY Max Planck Institut fur Physik, Munich, GERMANY

Moscow State University, Moscow, RUSSIA University of Salerno, Salerno, ITALY INFN - Sezione di Bari, ITALY INFN - Sezione di Bari, ITALY

Necmettin Erbakan University, Konya, TURKEY Middle East Technical University, Ankara, TURKEY University of Bari and INFN - Sezione di Bari, ITALY University of Torino, Torino, ITALY

University of Bari and INFN - Sezione di Bari, ITALY University of Pavia and INFN - Sezione di Pavia, ITALY

University of Bari and INFN - Sezione di Bari, ITALY Institute Jozef Stefan, Ljubljana, SLOVENIA

British University in Egypt, Cairo, EGYPT

Ecole Polytechnique - CNRS, Palaiseau, FRANCE MEPhI/CERN, Geneva, SWITZERLAND

University of Chinese Academy of Sciences, Beijing, CHINA INFN - Sezione di Genova, ITALY

University of Napoli and INFN - Sezione di Napoli, ITALY University of Bari and INFN - Sezione di Bari, ITALY Institute of Nuclear Physics, Cracow, POLAND INFN - Sezione di Pavia, ITALY

Goethe University, Frankfurt, GERMANY

Universitá della Calabria and INFN - Cosenza, ITALY University of Frankfurt and GSI, Frankfurt, GERMANY University of Trieste and INFN - Sezione di Trieste, ITALY

University of Parma and INFN - Milano Bicocca, ITALY University of Bari and INFN - Sezione di Bari, ITALY Shanghai JiaoTong University (SJTU), Shanghai, CHINA University of Vienna, Vienna, AUSTRIA Academia Sinica, Taipei, TAIWAN University of Torino, Torino, ITALY INFN - Sezione di Genova, ITALY Moscow State University, Moscow, RUSSIA 